\title{
New photometric observations of asteroids (1862) Apollo and (25143) Itokawa - an analysis of YORP effect
}

J. Ďurech ${ }^{1}$, D. Vokrouhlický ${ }^{1}$, M. Kaasalainen ${ }^{2}$, P. Weissman ${ }^{3}$, S. C. Lowry ${ }^{3}$, E. Beshore ${ }^{4}$, D. Higgins ${ }^{5}$, Y. N. Krugly ${ }^{6}$, V. G. Shevchenko ${ }^{6}$, N. M. Gaftonyuk ${ }^{7}$, Y.-J. Choi ${ }^{3,8}$, R. A. Kowalski ${ }^{4}$, S. Larson ${ }^{4}$, B. D. Warner ${ }^{9}$, A. L. Marshalkina ${ }^{10}$, M. A. Ibrahimov ${ }^{10}$, I. E. Molotov ${ }^{11}$, T. Michałowski ${ }^{12}$, and K. Kitazato ${ }^{13}$

\author{
1 Astronomical Institute, Charles University in Prague, V Holešovičkách 2, 18000 Prague, Czech Republic \\ e-mail: durech@sirrah.troja.mff.cuni.cz \\ 2 Department of Mathematics and Statistics, Rolf Nevanlinna Institute, PO Box 68, 00014 University of Helsinki, Finland \\ 3 Science Division, Jet Propulsion Laboratory, 4800 Oak Grove Drive, MS 183-301, Pasadena, CA 91109, USA \\ ${ }^{4}$ Lunar and Planetary Laboratory, University of Arizona, Tucson, AZ 85721-0092, USA \\ 5 Hunters Hill Observatory, 7 Mawalan Street, Ngunnawal ACT 2913, Australia \\ 6 Institute of Astronomy of Kharkiv National University, Sumska str. 35, Kharkiv 61022, Ukraine \\ 7 Crimean Astrophysical Observatory, Simeiz 98680, Ukraine \\ 8 Korea Astronomy \& Space Science Institute, 61-1 Hwa-am Dong, Yuseong Gu, Daejon 305-348, South Korea \\ 9 Palmer Divide Observatory, Colorado Springs, Colorado 80908, USA \\ ${ }_{10}$ Ulugh Beg Astronomical Institute, Uzbek Academy of Sciences, Astronomicheskaya 33, Tashkent 100052, Uzbekistan \\ 11 Keldysh Institute of Applied Mathematics, RAS, Miusskaya sq. 4, Moscow 125047, Russia \\ 12 Astronomical Observatory, Adam Mickiewicz University, Słoneczna 36, 60-286 Poznań, Poland \\ 13 Department of Earth and Planetary Science, The University of Tokyo, Bynkyo-ku, Tokyo 113-0033, Japan
}

Received 27 February 2008 / Accepted 18 May 2008

\section{ABSTRACT}

\begin{abstract}
Aims. Asteroid (1862) Apollo is one of two asteroids in which the YORP effect was detected. We carried out new photometric observations of Apollo in April 2007 to enlarge the time line and to derive a more precise shape and spin state model. We also observed another YORP-candidate, asteroid (25143) Itokawa, in December 2006 and January 2007 to obtain a longer time line. An estimation of the YORP strength on Itokawa based on its precise shape model from the Hayabusa mission predicted the deceleration to be already observable during the 2007 apparition.

Methods. We used the lightcurve inversion method to model the shape and spin state of Apollo. For Itokawa, the shape and pole direction are known to a high degree of accuracy from the Hayabusa mission, so we used a modified version of lightcurve inversion with only two free parameters - the rotation period and its linear change in time.

Results. The new model of Apollo confirms earlier results. The observed acceleration of Apollo's rotation rate is $(5.5 \pm 1.2) \times$ $10^{-8} \mathrm{rad} \mathrm{d}^{-2}$, which is in agreement with the theoretically predicted value. For Itokawa, the theoretical YORP value is sensitive to the resolution of the shape model and lies in the range from -2 to $-3 \times 10^{-7} \mathrm{rad} \mathrm{d}^{-2}$. This is inconsistent with results of lightcurve inversion that place an upper limit to the change of Itokawa's rotation rate $\sim 1.5 \times 10^{-7} \mathrm{rad} \mathrm{d}^{-2}$.
\end{abstract}

Key words. minor planets, asteroids - methods: data analysis - techniques: photometric

\section{Introduction}

The orbital and rotational dynamics of small asteroids is affected by the anisotropic reflection and thermal emission of sunlight from their surfaces. The corresponding net torque can change the spin states of asteroids. This so-called YORP (Yarkovsky-O'Keefe-Radzievskii-Paddack) effect was introduced by Rubincam (2000), who studied the evolution of asteroid spin states caused by thermal emission. The YORP effect can also explain the alignment of spin axes of Koronis family members (Slivan 2002; Vokrouhlický et al. 2003). The first direct detection of YORP was achieved on asteroids (1862) Apollo (Kaasalainen et al. 2007) and (54509) YORP (Lowry et al. 2007; Taylor et al. 2007). Kitazato et al. (2007) reported the direct detection of YORP on asteroid (25143) Itokawa but they later rescinded their result.

In this paper we present new photometric observations of asteroids Apollo and Itokawa. For Apollo (Sect. 2), the new data are fully consistent with the previous physical model published by Kaasalainen et al. (2007). For Itokawa (Sect. 3), we show that a constant-period model gives the best fit to the lightcurves. We compare theoretical YORP values with those obtained by lightcurve inversion and show that the YORP deceleration predicted by theory is inconsistent with observations. We also show that the possible change of Itokawa's rotation state that occurred during its close encounter with the Earth in June 2004 is likely too small to explain the discrepancy between theory and observations.

\section{2. (1862) Apollo}

Apollo was one of the two first asteroids in which the YORP effect was detected. Kaasalainen et al. (2007) used a set of 39 photometric lightcurves from four apparitions in 1980-2005 and showed that it could not be fitted with a model with a constant rotation period. However, when a linear change in 
Table 1. Aspect data for new observations of Apollo. The table lists Apollo's distance from the Sun $r$ and from the Earth $\Delta$, the solar phase angle $\alpha$, the geocentric ecliptic coordinates of the asteroid $(\lambda, \beta)$, and the observatory ( $\mathrm{HH}$ - Hunters Hill Observatory, $35 \mathrm{~cm}$; K - Kharkiv Observatory, $70 \mathrm{~cm}$; S - Simeiz Observatory, $1 \mathrm{~m}$; M - Maidanak Observatory, $60 \mathrm{~cm}$ ).

\begin{tabular}{|c|c|c|c|c|c|c|}
\hline Date & $\begin{array}{c}r \\
{[\mathrm{AU}]}\end{array}$ & $\begin{array}{c}\Delta \\
{[\mathrm{AU}]}\end{array}$ & $\begin{array}{c}\alpha \\
{[\mathrm{deg}]}\end{array}$ & $\begin{array}{c}\lambda \\
{[\mathrm{deg}]}\end{array}$ & $\begin{array}{c}\beta \\
{[\operatorname{deg}]}\end{array}$ & Obs. \\
\hline 20070409.6 & 1.282 & 0.343 & 30.5 & 239.8 & 2.7 & $\overline{\mathrm{HH}}$ \\
\hline 20070410.6 & 1.273 & 0.331 & 30.4 & 240.3 & 2.5 & $\mathrm{HH}$ \\
\hline 20070411.5 & 1.265 & 0.320 & 30.4 & 240.8 & 2.3 & $\mathrm{HH}$ \\
\hline 20070412.0 & 1.261 & 0.314 & 30.4 & 241.1 & 2.2 & $\mathrm{~K}$ \\
\hline 20070412.7 & 1.255 & 0.307 & 30.4 & 241.5 & 2.1 & $\mathrm{HH}$ \\
\hline 20070412.9 & 1.252 & 0.303 & 30.3 & 241.6 & 2.0 & M \\
\hline 20070413.0 & 1.252 & 0.302 & 30.3 & 241.7 & 2.0 & K \\
\hline 20070414.7 & 1.237 & 0.283 & 30.3 & 242.7 & 1.6 & $\mathrm{HH}$ \\
\hline 20070414.9 & 1.235 & 0.280 & 30.3 & 242.9 & 1.6 & M \\
\hline 20070415.0 & 1.234 & 0.279 & 30.4 & 243.0 & 1.5 & K \\
\hline 20070416.0 & 1.225 & 0.267 & 30.4 & 243.7 & 1.3 & $S$ \\
\hline 20070417.6 & 1.210 & 0.248 & 30.6 & 244.9 & 0.8 & $\mathrm{HH}$ \\
\hline 20070418.6 & 1.201 & 0.237 & 30.7 & 245.8 & 0.4 & $\mathrm{HH}$ \\
\hline 20070418.9 & 1.198 & 0.234 & 30.8 & 246.0 & 0.3 & M \\
\hline 20070419.0 & 1.197 & 0.233 & 30.8 & 246.1 & 0.3 & $\mathrm{~S}$ \\
\hline 20070419.7 & 1.191 & 0.226 & 30.9 & 246.7 & 0.0 & $\mathrm{HH}$ \\
\hline 20070419.9 & 1.189 & 0.223 & 31.0 & 246.9 & -0.1 & M \\
\hline 20070420.0 & 1.188 & 0.221 & 31.0 & 247.1 & -0.1 & $\mathrm{~S}$ \\
\hline 20070420.6 & 1.183 & 0.215 & 31.2 & 247.6 & -0.4 & $\mathrm{HH}$ \\
\hline
\end{tabular}

rotational frequency was assumed, the data were fitted down to the noise level. Moreover, the value of the YORP acceleration derived from the lightcurve inversion agreed very well with the value computed numerically from the convex shape model and the spin axis orientation - making the whole model physically self consistent. The rotation parameters derived by Kaasalainen et al. (2007) were as follows: ecliptic coordinates of the spin axis direction $\lambda=50^{\circ}, \beta=-71^{\circ}$ (with an error $\pm 7^{\circ}$ of arc), the sidereal rotation period $P=3.065447 \pm 0.000003 \mathrm{~h}$ for JD 2444557.0 , and the acceleration of the rotation speed $v \equiv \mathrm{d} \omega / \mathrm{d} t=(5.3 \pm 1.3) \times 10^{-8} \mathrm{rad} \mathrm{d}^{-2}$.

\subsection{New observations}

Apollo was in a favorable apparition in April 2007 and we carried out new photometric observations to find out if the model published by Kaasalainen et al. (2007) is consistent with the new data. The details of the observing geometries for 15 new lightcurves are given in Table 1.

\subsection{Model}

New observations were combined with the old data set and were analyzed using the lightcurve inversion method (Kaasalainen et al. 2001, 2003b; Kaasalainen \& Ďurech 2007). The new physical model is very close to the previous one: $\lambda=48^{\circ}$, $\beta=-72^{\circ}, P=3.065448 \pm 0.000003 \mathrm{~h}$ for JD 2444557.0 (13.5 November 1980), and $v=(5.5 \pm 1.2) \times 10^{-8} \mathrm{rad} \mathrm{d}^{-2}$. The uncertainty of the derived parameters remains almost the same due to the fact that we have added only two years to the total time interval of 25 years (the first photometry of Apollo is from 1980, see Kaasalainen et al. 2007 for details). However, contrary to the photometric data used by Kaasalainen et al. (2007), two lightcurves from 1998 are now not crucial for the YORP detection. If we exclude them from the data set, the best solution with $v=7 \times 10^{-8} \mathrm{rad} \mathrm{d}^{-2}$ still gives $\sim 10 \%$ lower $\chi^{2}$ than a constant-period model with $v=0$. This is an important consistency check of YORP detection on Apollo.

The convex shape model of Apollo is shown in Fig. 1 and two representative lightcurves and corresponding fits are shown in Fig. 2. The derived shape model together with the complete lightcurve data set is available at astro . troja.mff.cuni.cz/ projects/asteroids3D. The dimension along the $z$-axis is not well constrained from relative photometry. Thus the real shape may not be as "flat" as the model in Fig. 1.

We used the derived shape model and spin solution and computed the theoretical YORP strength numerically (Vokrouhlický \& Čapek 2002; Čapek \& Vokrouhlický 2004, 2005). For a bulk density $\rho=2.7 \mathrm{~g} \mathrm{~cm}^{-3}$, an effective diameter $D=1.45 \mathrm{~km}$, Bond albedo 0.1 , and thermal conductivity $K=0.02 \mathrm{~W} \mathrm{~m}^{-1} \mathrm{~K}^{-1}$, the predicted value of YORP acceleration is $\mathrm{d} \omega / \mathrm{d} t=8.0 \times$ $10^{-8} \mathrm{rad} \mathrm{d}^{-2}$. which is in good agreement with the measured value. The estimation of the bulk density is based on results of Chesley et al. (2003), who detected the Yarkovsky effect on asteroid (6489) Golevka and derived the bulk density of Golevka to $2.7 \mathrm{~g} \mathrm{~cm}^{-3}$. Because both Apollo and Golevka are Q-type asteroids, we used this value of the bulk density. The value of the effective diameter was taken from Harris (1998).

The YORP strength is proportional to $\left(\rho D^{2}\right)^{-1}$ so making the theoretical value equal to the observed one is possible by changing the diameter or density slightly. For example, if we assume the bulk density to be $\rho=2.7 \mathrm{~g} \mathrm{~cm}^{-3}$ and the effective diameter to be $D=1.75 \mathrm{~km}$, then the predicted rotation rate acceleration is exactly the observed value $5.5 \times 10^{-8} \mathrm{rad} \mathrm{d}^{-2}$.

As was already stated by Kaasalainen et al. (2007), the numerically derived YORP strength for Apollo was stable against slight variations of the shape model. The numerically derived acceleration rate varied with different shape variants from the lightcurve inversion - different shape representation and optimization procedures provided us with slightly different shapes that produced almost the same lightcurves but the YORP strength for them differed by a few tens of percent. We produced several variants of shape (all giving a satisfactory fit to photometric data) and the computed values of $\mathrm{d} \omega / \mathrm{d} t$ again varied within a few tens of percent. These variants did not introduce surface irregularities below the resolution of the lightcurve model.

\section{3. (25143) Itokawa}

Asteroid Itokawa was the subject of an extensive observing campaign during its 2001 and 2004 apparitions. A physical model of Itokawa was derived from its photometric lightcurves by Kaasalainen et al. (2003a, 2008). No YORP effect was detected and the derived spin state and global shape agreed well with the real shape revealed by the Hayabusa mission (Fujiwara et al. 2006; Abe et al. 2006). Recently, the detection of the deceleration of the rotation of Itokawa was published by Kitazato et al. (2007). However, their results were affected by a slightly incorrect initial orientation of Itokawa in space. After correcting this error, the decelerating model did not fit the data better than a constant-period model and there was no evidence of YORP (Kitazato et al. at DPS meeting 2007).

\subsection{New observations}

We carried out new photometric observations of Itokawa during its 2006/07 apparition. Details are given in Table 2. Together with five new lightcurves and those by Kitazato et al. (2007), 


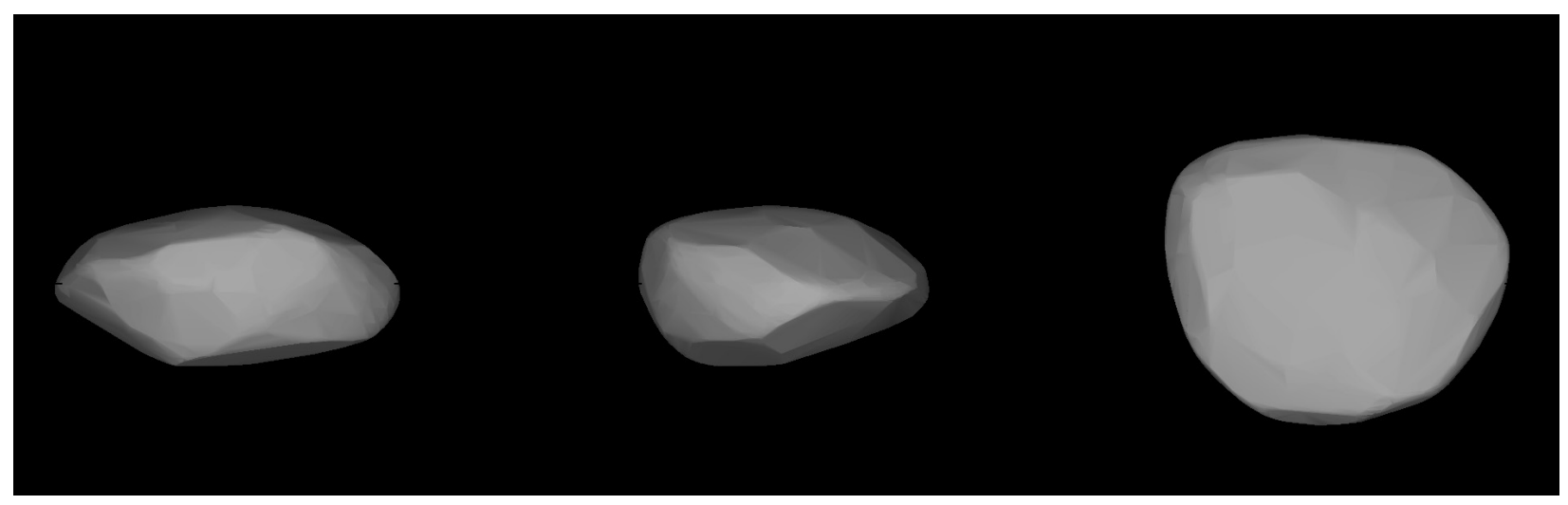

Fig. 1. The shape model of Apollo shown from equatorial level (left and centre, $90^{\circ}$ apart) and pole-on (right).
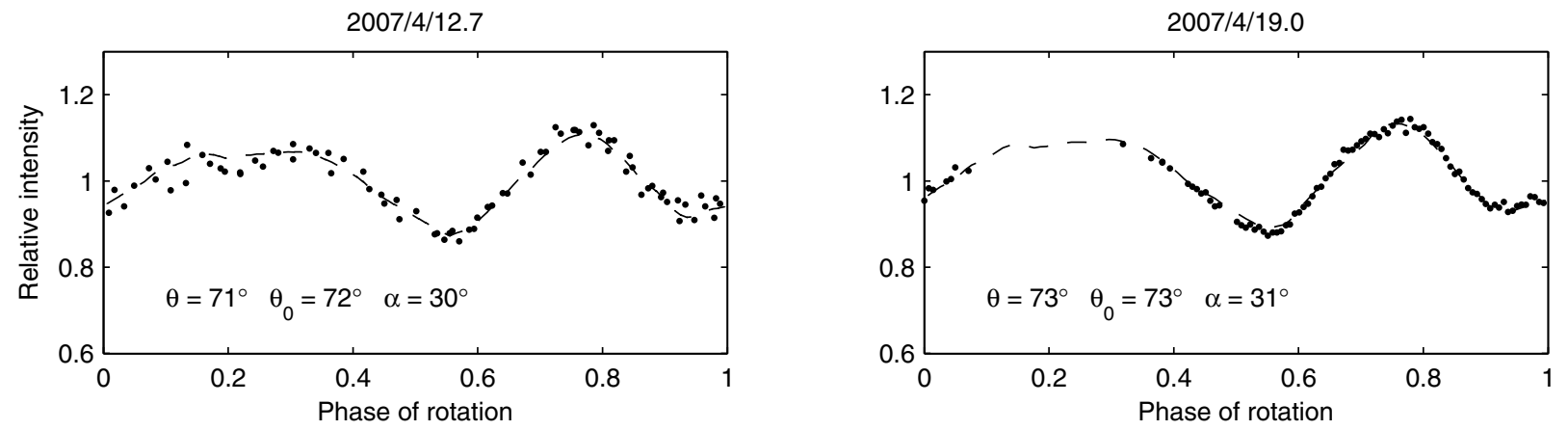

Fig. 2. Examples of Apollo's lightcurves from the 2007 apparition fitted with our model (dashed curve). The viewing and illumination geometry is given by the aspect angle $\theta$, the solar aspect angle $\theta_{0}$, and the solar phase angle $\alpha$.

Table 2. Aspect data for new observations of Itokawa. The table lists asteroid distance from the Sun $r$, from the Earth $\Delta$, the solar phase angle $\alpha$, the geocentric ecliptic coordinates of the asteroid $(\lambda, \beta)$, and the observatory (ML - Mt. Lemmon, 1.5 m, MB - Mt. Bigelow, $1.52 \mathrm{~m}$ ).

\begin{tabular}{ccccccc}
\hline \hline Date & $\begin{array}{c}r \\
{[\mathrm{AU}]}\end{array}$ & $\begin{array}{c}\Delta \\
{[\mathrm{AU}]}\end{array}$ & $\begin{array}{c}\alpha \\
{[\mathrm{deg}]}\end{array}$ & $\begin{array}{c}\lambda \\
{[\mathrm{deg}]}\end{array}$ & $\begin{array}{c}\beta \\
{[\mathrm{deg}]}\end{array}$ & Obs. \\
\hline 20061225.3 & 1.507 & 0.538 & 10.6 & 109.5 & 2.3 & ML \\
20061226.3 & 1.504 & 0.532 & 9.7 & 109.1 & 2.3 & ML \\
20070115.3 & 1.435 & 0.464 & 11.1 & 98.6 & 3.3 & ML \\
20070124.2 & 1.401 & 0.459 & 20.5 & 93.9 & 3.5 & MB \\
20070125.2 & 1.397 & 0.460 & 21.6 & 93.5 & 3.5 & MB \\
\hline
\end{tabular}

the whole data set contains 80 lightcurves observed between December 2000 and January 2007.

\subsection{Model}

First, we used a modified version of the lightcurve inversion method where the rotation period and a linear change of the rotation rate were the only free parameters. All other parameters were assumed to be known and were fixed during the optimization. We used the shape model of Itokawa published by Gaskell et al. (2006) ${ }^{1}$ for our analysis and reduced it to 10000 surface facets. This reduction had almost no effect on synthetic lightcurves but significantly reduced the time needed for computations. The orientation of the model with respect to the Earth and the Sun was computed from its initial orientation values (J2000): the pole direction in ecliptic coordinates $\lambda=269.03^{\circ}, \beta=-89.53^{\circ}$ with an uncertainty of $0.005^{\circ}$ of arc

\footnotetext{
${ }^{1}$ Models are available at http://hayabusa.sci.isas.jaxa.jp.
}

(Gaskell et al. 2006; Demura et al. 2006), and the prime meridian $129.73^{\circ}$. The prime meridian for $\mathbf{J} 2000$ was derived from the orientation of Itokawa as measured by Hayabusa during the encounter in 2005 using the fixed rotation rate $712.1437611 \mathrm{deg} / \mathrm{d}$ (Gaskell, personal communication). We used Hapke's scattering model with the same parameters as Kitazato et al. (2007): the single-particle scattering albedo $\varpi=0.4$, the asymmetry parameter of the single-particle phase function $g=-0.35$, the opposition surge amplitude $B_{0}=0.89$ and width $h=0.01$, and the macroscopic roughness $\theta=26^{\circ}$. These values are consistent with those determined by Lederer et al. (2005) from discintegrated photometry. With only two free parameters ( $P$ and $v)$, we searched for the best fit between observed and modelled lightcurves. Observed lightcurves were treated as relative, which means that they were vertically shifted to give the best match with the synthetic curves.

Our results at this stage show that there is no signature of YORP in the dataset. The model with formally constant period fits all data as well as the model with a small nonzero value of $v$. The apparent best solution gives $P=12.1323 \pm 0.0002 \mathrm{hr}$ and $v=(1.5 \pm 15) \times 10^{-8} \mathrm{rad} \mathrm{d}^{-2}$. We can place only an upper limit on the YORP strength $|v| \lesssim 1.5 \times 10^{-7} \mathrm{rad} \mathrm{d}^{-2}$ (corresponding to a $10 \%$ increase in $\chi^{2}$ ). If $|v|$ were larger, phase shifts for a constant-period model would be detectable.

\subsection{Theoretical YORP value}

We also computed the theoretical YORP value numerically using the same numerical code as for Apollo (Vokrouhlický \& Čapek 2002; Čapek \& Vokrouhlický 2004, 2005). We adopted a surface thermal inertia $\Gamma=750 \mathrm{~J} \mathrm{~m}^{-2} \mathrm{~s}^{-0.5} \mathrm{~K}^{-1}$ 
Table 3. Numerically computed values of $\mathrm{d} \omega / \mathrm{d} t$ for shape models of Itokawa with different resolutions (number of surface triangular facets). The boldfaced models with 49152 and 196608 facets are the original low-resolution shape models available at http://hayabusa.sci . isas. jaxa.jp. All other models in the table were derived from these two models.

\begin{tabular}{rlrl}
\hline \hline Resolution & $\begin{array}{l}\mathrm{d} \omega / \mathrm{d} t \\
\left(\times 10^{-7} \mathrm{rad} \mathrm{d}^{-2}\right)\end{array}$ & Resolution & $\begin{array}{l}\mathrm{d} \omega / \mathrm{d} t \\
\left(\times 10^{-7} \mathrm{rad} \mathrm{d}^{-2}\right)\end{array}$ \\
\hline 1000 & -2.779 & 40000 & -1.886 \\
2000 & -0.750 & 49152 & -2.478 \\
3000 & -1.053 & $\mathbf{4 9 1 5 2}$ & $-\mathbf{2 . 1 0 5}$ \\
4000 & -0.730 & 60000 & -2.749 \\
5000 & -1.134 & 80000 & -2.999 \\
6000 & -1.038 & 100000 & -2.940 \\
7000 & -1.318 & 120000 & -2.944 \\
8000 & -1.457 & 140000 & -2.860 \\
9000 & -1.440 & 160000 & -2.914 \\
10000 & -1.365 & 180000 & -3.021 \\
20000 & -2.022 & $\mathbf{1 9 6 6 0 8}$ & $-\mathbf{3 . 0 9 7}$ \\
30000 & -2.059 & & \\
\hline
\end{tabular}

(Müller et al. 2005), and used pole and shape models of Gaskell et al. (2006). While unable to compute YORP for the highestresolution versions, we did so for the lower-resolution variants of the original model as well as for a number of synthetic models with reduced resolution (see Table 3 ). We also used Itokawa's mass of $3.58 \times 10^{10} \mathrm{~kg}$ determined by the Hayabusa spacecraft (Abe et al. 2006). Our computations always include self-shadowing of different surface elements, an effect which is particularly important for Itokawa's rugged shape and its high value of obliquity. We use Lambert's isotropic emission at thermal wavelengths.

Our results indicate that theoretical YORP values are highly sensitive to the shape-model resolution, thus confirming the earlier finding of Scheeres et al. (2007). Even with the finest model we used, the predicted YORP value did not converge exactly, thus leaving some uncertainty in the result. We note that this is in agreement with the theoretical prediction of Nesvorný \& Vokrouhlický $(2007,2008)$ whose analytical calculations show that the YORP value may depend on very small-scale irregularities of the asteroid's shape. Moreover, not only the resolution but also the representation of the shape for a given resolution plays an important role - even models with the same resolution give different results (see Table 3). Clearly, theoretical modelling of YORP still needs improvements in the future and Itokawa - with its detailed shape model - is an ideal object for comparing theoretically predicted YORP values with observations.

Scheeres et al. (2007) derived an expected YORP for Itokawa from a theoretical model. They predicted the deceleration in the spin rate to be in the range from -2.5 to $-4.5 \times 10^{-17} \mathrm{rad} \mathrm{s}^{-2}$ (which translates to -1.9 to $-3.4 \times 10^{-7} \mathrm{rad} \mathrm{d}^{-2}$ ). These values agree well with ours (Table 3 ) and the computational methodologies are consistent with each other.

However, the predicted deceleration rate $\sim 3 \times 10^{-7} \mathrm{rad} \mathrm{d}^{-2}$ is in contradiction with observations. Observed lightcurves cannot be fitted well for $|v| \gtrsim 1.5 \times 10^{-7} \mathrm{rad} \mathrm{d}^{-2}$. For example, for a fixed value $v=-2 \times 10^{-7} \mathrm{rad} \mathrm{d}^{-2}$, the discrepancy between observed and synthetic lightcurves is significant, giving a $\chi^{2}$ value about $20 \%$ higher than for $v=0$.

\subsection{Close encounter with the earth}

To explain the inconsistency between theoretically predicted YORP values and observations that do not show any evidence of YORP, we assumed that Itokawa's rotation state could have been affected by its close encounter with the Earth in June 2004. According to Scheeres et al. (2000), the maximum change $\delta \omega$ of an asteroid's rotation rate during its planetary flyby can be estimated as

$|\delta \omega| \simeq \frac{1}{2} \frac{B-A}{C} \frac{G M}{q^{2} v_{\infty}}$,

where $A \leq B \leq C$ are proper values of asteroid's inertia tensor, $G$ is the gravitational constant, $M$ is the mass of the planet, $q$ is the minimum asteroid-planet distance during the encounter, and $v_{\infty}$ is the relative velocity at a large distance. For Itokawa, $(B-A) / C \simeq 0.66, q \simeq 0.0129 \mathrm{AU}$, and $v_{\infty} \simeq 6.1 \mathrm{~km} \mathrm{~s}^{-1}$. Thus the estimated maximum change in Itokawa's rotation rate is $\sim 5 \times 10^{-4} \mathrm{rad} \mathrm{d}^{-1}$ (see also Vokrouhlický et al. 2004), which could cause a phase shift of lightcurves of the same order as the phase shift caused by YORP. For example, the phase shift due to the encounter accumulated within three years (2004-2007 time interval $t \simeq 3 \mathrm{yr}, \delta \omega \simeq 5 \times 10^{-4} \mathrm{rad} \mathrm{d}^{-1}$ ) is $\delta \phi_{\mathrm{enc}}=\delta \omega \Delta t \simeq 31^{\circ}$. This is almost the same as the phase shift due to YORP with $v \simeq 2 \times 10^{-7} \mathrm{rad} \mathrm{d}^{-2}$ (see Sect. 3.3) in six years 2001-2007, $\delta \phi_{\mathrm{YORP}}=0.5 v \Delta t^{2} \simeq 27^{\circ}$.

For instance, a formal solution with $\delta \omega=1.5 \times 10^{-4} \mathrm{rad} \mathrm{d}^{-1}$ would allow $v=-1.9 \times 10^{-7} \mathrm{rad} \mathrm{d}^{-2}$ still to be consistent with observations, giving only an $\sim 5 \%$ higher $\chi^{2}$ than the formal best fit.

However, Eq. (1) neglects asteroid rotation during the encounter (see Scheeres et al. 2000, for details) and cannot be used for Itokawa's encounter in 2004, which was not fast enough with respect to the rotation period of Itokawa. When we take asteroid rotation into account, the total change of rotation rate $\delta \omega$ can be derived by integrating the torque acting on the asteroid along the hyperbolic trajectory:

$\delta \omega=3 \frac{G M}{a^{3}} \frac{B-A}{C} \int_{-\infty}^{\infty}\left(\frac{a}{r}\right)^{3} n_{x} n_{y} \mathrm{~d} t$,

where $a$ is the semimajor axis of the trajectory, $r$ is the changing distance between the Earth and the asteroid, and $n_{x}=\boldsymbol{n} \cdot \boldsymbol{e}_{x}$, $n_{y}=\boldsymbol{n} \cdot \boldsymbol{e}_{y}$ are projections of unit vectors $\boldsymbol{e}_{x}, \boldsymbol{e}_{y}$ corresponding to eigenvectors of the inertia tensor in the direction $\boldsymbol{n}$ towards the Earth. Direct numerical integration of Eq. (2) for Itokawa's encounter in 2004 yields $\delta \omega=-5.3 \times 10^{-7} \mathrm{rad} \mathrm{d}^{-1}$, which is also almost the maximum possible value for any initial rotation phase of Itokawa with respect to the Earth. This value is too small to have any observable effect on lightcurves. Thus the inconsistency between theory and observations cannot be explained by this model of close-encounter gravitational interaction.

Our simplified model assumes that Itokawa is a rigid body. Any effect of nonrigidity would change our estimation based on Eq. (2). For example, to obtain the desired change of rotation rate $\delta \omega \sim 1.5 \times 10^{-4} \mathrm{rad} \mathrm{d}^{-1}$ that would allow values of $\mathrm{d} \omega / \mathrm{d} t$ to be consistent with theory, one needs the relative change of the moment of inertia $\delta C / C \sim \delta \omega / \omega \sim 10^{-5}$. However, we have not carried out any estimations of whether this change of the moment of inertia during the encounter is physically feasible.

\section{Conclusions}

New photometric observations of Apollo confirmed the physical model derived by Kaasalainen et al. (2007). The detected 
$2001 / 1 / 24.5$

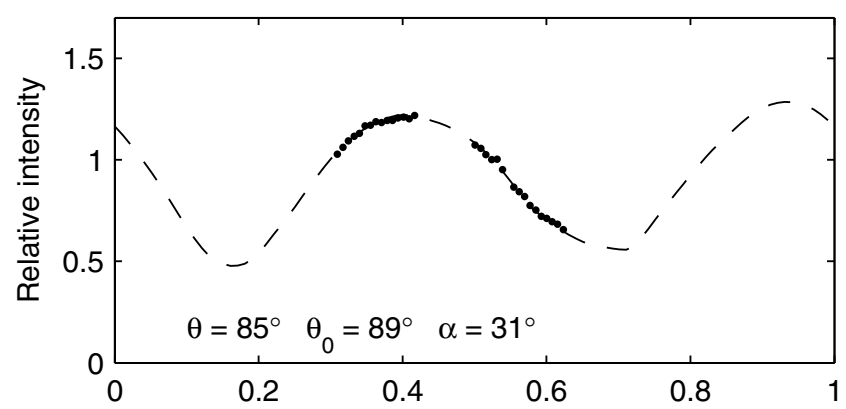

2004/7/16.3

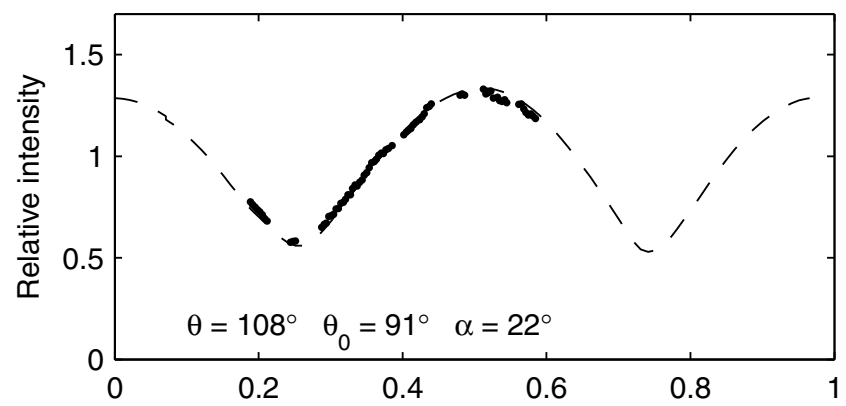

2007/1/15.3

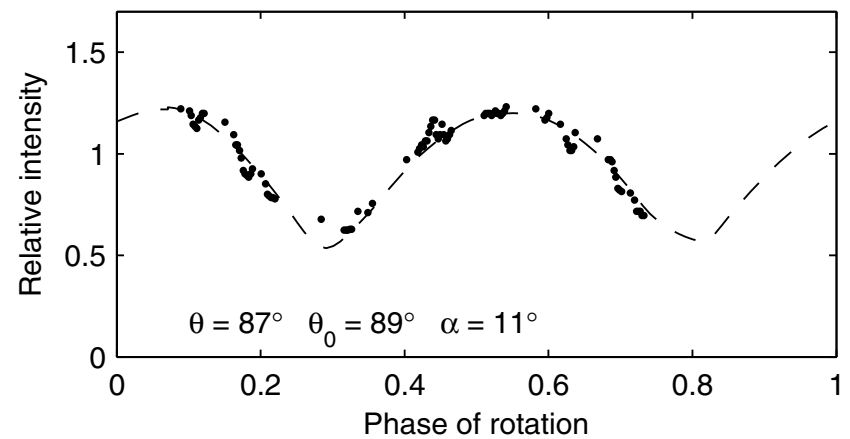

2004/7/7.3

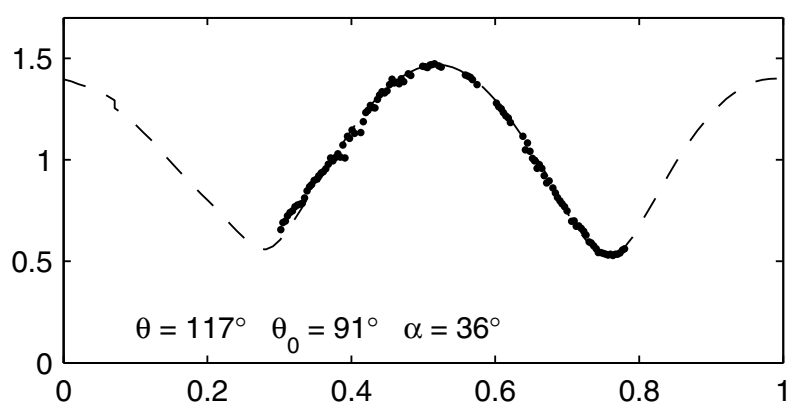

$2006 / 12 / 25.3$

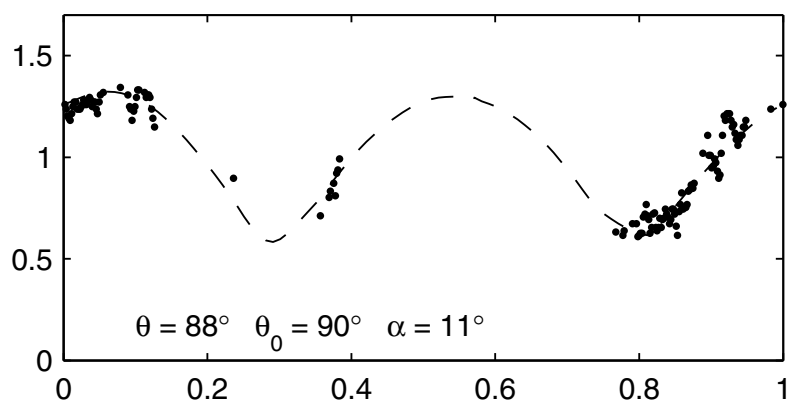

2007/1/24.2

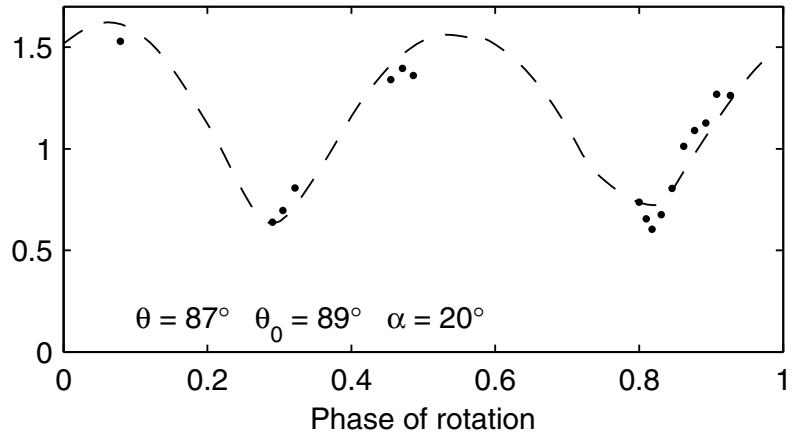

Fig. 3. Examples of Itokawa's lightcurves fitted with synthetic lightcurves based on the Hayabusa shape model ( 10000 surface facets, dashed curve) rotating with a constant period $P=12.13237 \mathrm{~h}$. The viewing and illumination geometry is given by the aspect angle $\theta$, the solar aspect angle $\theta_{0}$, and the solar phase angle $\alpha$. Small-scale brightness variations in lightcurves from 2006/12/25.3 and 2007/1/15.3 are artifacts of CCD image reduction - Itokawa moved close to the galactic plane and crossed background stars.

acceleration rate is fully consistent with the value predicted by theory. Moreover, our work relaxed a crucial dependence of the YORP detection for Apollo on the 1998 lightcurve data. YORP on Apollo is dominated by its global shape features - slightly different shape models give YORP values that differ only within a few tens of percent. Further observations will reduce the uncertainty of $v$. The next favorable apparition of Apollo is in 2009/2010.

A thorough analysis of Itokawa's lightcurves with the use of a precise shape model and spin state derived from the Hayabusa mission did not reveal any signature of deviation from a constant-period rotation. Numerical simulations give different predictions of $\mathrm{d} \omega / \mathrm{d} t$ depending on what resolution of the shape model is used. However, the systematically high negative values of $\mathrm{d} \omega / \mathrm{d} t$ are not consistent with observations. The question of whether this inconsistency is caused by some imperfections in the numerical computations of the YORP effect or by, e.g., some effects of nonrigidity of Itokawa during its close encounter in 2004 remains open. It is necessary to carry out more observations of Itokawa during the next apparition(s) to detect possible deviations from a constant-period model caused by YORP. Itokawa will be observable again in December 2009 and December 2012. During both apparitions, it will reach $\sim 19 \mathrm{mag}$ and will move close to the galactic plane. An estimated detection limit for YORP when lightcurves from 2009 and 2012 will be available is $\sim 7 \times 10^{-8} \mathrm{rad} \mathrm{d}^{-2}$ and $\sim 4 \times 10^{-8} \mathrm{rad} \mathrm{d}^{-2}$, respectively.

The lightcurve inversion method can easily include the YORP effect as a linear term in calculations of the change of the rotation rate with time. This technique will likely lead to other YORP detections in the future - either by enlarging the time line of observations for larger asteroids with archived observations from past decades or by a dedicated photometric survey of small, faster evolving objects.

Acknowledgements. The work of J.D. and D.V. was supported by grants GACR 205/07/P070 and GACR 205/08/0064 of the Czech grant agency and by the Research Program MSM0021620860 of the Ministry of Education. The work of M.K. was supported by the Academy of Finland. Y.N.K. was partly supported by the Main Astronomical Observatory of National Academy of Science of Ukraine. 
This work was supported in part by the NASA Planetary Astronomy Program and was performed in part at Queens University Belfast and the Jet Propulsion Laboratory under a contract with NASA. SCL also acknowledges support from the Leverhulme Trust. The work of T. M. was supported by the Polish Ministry of Science and Higher Education - grant N N203 302535. We thank the anonymous referee for important comments.

\section{References}

Abe, S., Mukai, T., Hirata, N., et al. 2006, Science, 312, 1344

Čapek, D., \& Vokrouhlický, D. 2004, Icarus, 172, 526

Čapek, D., \& Vokrouhlický, D. 2005, in IAU Coll., 197, Dynamics of Populations of Planetary Systems, ed. Z. Knežević, \& A. Milani, 171

Chesley, S. R., Ostro, S. J., Vokrouhlický, D., et al. 2003, Science, 302, 1739

Demura, H., Kobayashi, S., Nemoto, E., et al. 2006, Science, 312, 1347

Fujiwara, A., Kawaguchi, J., Yeomans, D. K., et al. 2006, Science, 312, 1330

Gaskell, R., Barnouin-Jha, O., Scheeres, D., et al. 2006, in Paper AIAA-20066660, AIAA/AAS Astrodynamics Specialist Meeting, Keystone, Colorado, August 2006

Harris, A. W. 1998, Icarus, 131, 291

Kaasalainen, M., \& Durech, J. 2007, in Near Earth Objects, our Celestial Neighbors: Opportunity and Risk, ed. A. Milani, G. B. Valsecchi, \& D. Vokrouhlický (Cambridge: Cambridge University Press), 151
Kaasalainen, M., Torppa, J., \& Muinonen, K. 2001, Icarus, 153, 37 Kaasalainen, M., Kwiatkowski, T., Abe, M., et al. 2003a, A\&A, 405, L29 Kaasalainen, M., Durech, J., Warner, B. D., Krugly, Y. N., \& Gaftonyuk, N. M. 2007, Nature, 446, 420

Kaasalainen, M., Abe, M., Byron, J., et al. 2008, in ASP Conf. Ser., ed. H. Yano, A. Fujiwara, D. Yeomans, \& M. Zolensky, in press

Kaasalainen, S., Piironen, J., Kaasalainen, M., et al. 2003b, Icarus, 161, 34

Kitazato, K., Abe, M., Ishiguro, M., \& Ip, W.-H. 2007, A\&A, 472, L5

Lederer, S. M., Domingue, D. L., Vilas, F., et al. 2005, Icarus, 173, 153

Lowry, S. C., Fitzsimmons, A., Pravec, P., et al. 2007, Science, 316, 272

Müller, T. G., Sekiguchi, T., Kaasalainen, M., Abe, M., \& Hasegawa, S. 2005, A\&A, 443, 347

Nesvorný, D., \& Vokrouhlický, D. 2007, AJ, 134, 1750

Nesvorný, D., \& Vokrouhlický, D. 2008, AJ, 136, 291

Rubincam, D. P. 2000, Icarus, 148, 2

Scheeres, D. J., Ostro, S. J., Werner, R. A., Asphaug, E., \& Hudson, R. S. 2000, Icarus, 147, 106

Scheeres, D. J., Abe, M., Yoshikawa, M., et al. 2007, Icarus, 188, 425

Slivan, S. M. 2002, Nature, 419, 49

Taylor, P. A., Margot, J.-L., Vokrouhlický, D., et al. 2007, Science, 316, 274

Vokrouhlický, D., \& Čapek, D. 2002, Icarus, 159, 449

Vokrouhlický, D., Nesvorný, D., \& Bottke, W. F. 2003, Nature, 425, 147

Vokrouhlický, D., Čapek, D., Kaasalainen, M., \& Ostro, S. J. 2004, A\&A, 414, L21 